\title{
Menelaus' theorem, Clifford configurations and inversive geometry of the Schwarzian KP hierarchy
}

\author{
B.G. Konopelchenkoli and W.K. Schief \\ School of Mathematics, The University of New South Wales, \\ Sydney, NSW 2052, Australia
}

\begin{abstract}
It is shown that the integrable discrete Schwarzian KP (dSKP) equation which constitutes an algebraic superposition formula associated with, for instance, the Schwarzian KP hierarchy, the classical Darboux transformation and quasi-conformal mappings encapsulates nothing but a fundamental theorem of ancient Greek geometry. Thus, it is demonstrated that the connection with Menelaus' theorem and, more generally, Clifford configurations renders the dSKP equation a natural object of inversive geometry on the plane. The geometric and algebraic integrability of dSKP lattices and their reductions to lattices of Menelaus-Darboux, Schwarzian $\mathrm{KdV}$, Schwarzian Boussinesq and Schramm type is discussed. The dSKP and discrete Schwarzian Boussinesq equations are shown to represent discretizations of families of quasi-conformal mappings.
\end{abstract}

\section{Introduction}

The celebrated Kadomtsev-Petviashvili (KP) equation was derived some thirty years ago [1] in connection with the propagation of weakly two-dimensional waves in nonlinear media. Subsequently [2, 3], it was shown that the KP equation is amenable to the Inverse Scattering Transform (IST) method and hence possesses all the remarkable properties which are commonly associated with

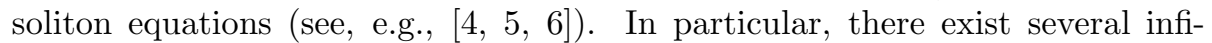
nite families of soliton equations which are related to the KP equation such as the KP, modified KP (mKP) and Schwarzian KP (SKP) hierarchies. Since its discovery, the KP equation has been the subject of extensive research. Thus, the KP equation and associated structures not only find application in plasma physics and hydrodynamics but also make important appearances in various other areas of physics and mathematics such as modern string theory [7, 8, 9] and algebraic geometry [10].

In the present paper, it is demonstrated that there exist profound connections between the KP hierarchy of modern soliton theory and beautiful constructions of plane geometry associated with the names of the Greek scholar Menelaus

\footnotetext{
${ }^{\dagger}$ Permanent address: Dipartimento di Fisica, Università di Lecce and Sezione INFN, 73100 Lecce, Italy
} 
(Menelaos of Alexandria, $\mathrm{I}^{\text {st }}-\mathrm{II}^{\text {nd }}$ century) who is perhaps best known for his treatise Sphaerica on spherical geometry and the distinguished British philosopher and mathematician Clifford (XIX ${ }^{\text {th }}$ century). Specifically, it is shown that the algebraic 6-point superposition formula for the scalar SKP hierarchy encapsulates nothing but the classical Theorem of Menelaus and, more generally, Clifford's point-circle configurations. As noticed by Clifford, the latter are naturally embedded in the classical theory of inversive geometry and, indeed, the 6-point relation, which is known to represent a discrete Schwarzian KP (dSKP) equation, turns out to be invariant under the group of inversive transformations. Thus, the dSKP equation constitutes a natural object of inversive geometry on the plane. By construction, the SKP hierarchy represents an infinite set of motions of points on the plane which preserve the basic dSKP 6-point relation and therefore its geometric properties.

In Section 2, we demonstrate the universal nature of the dSKP 6-point relation by (re-)deriving it in various different settings. Contact is made with the classical Darboux transformation and quasi-conformal mappings. In Section 3, the connection with Menelaus' theorem, Clifford configurations and inversive geometry is established. Section 4 is concerned with the interpretation of the dSKP equation as a lattice equation. It is shown how a canonical 'Lax triad' is obtained geometrically by introducing shape factors which encode certain angles in the dSKP lattice. It turns out that the shape factors are governed by an integrable discrete KP wave function equation. If the shape factors are real then the dSKP lattice consists of Menelaus figures. This observation leads to a Menelaus-type reduction of the discrete Darboux system defining special conjugate lattices in ambient spaces of arbitrary dimension. In Section 5, canonical dimensional reductions of the dSKP equation to the discrete Schwarzian Korteweg-de Vries (dSKdV) and Boussinesq (dSBQ) equations are interpreted geometrically. Degenerate Clifford lattices are shown to include discrete conformal mappings and the particular class of Schramm circle patterns. Lattices on circles and a Combescure-type transformation are considered. The final section deals with the natural continuum limit of the dSKP equation and its dSKdV and dSBQ reductions. Conformal and particular classes of quasi-conformal mappings are obtained.

The results presented here lead to the remarkable observation that, in principle, the complete KP theory may be retrieved from an elementary theorem of plane geometry which is 2000 years old!

\section{The discrete Schwarzian KP equation}

The present paper is concerned with the soliton-theoretic and geometric significance of the 6-point relation

$$
\frac{\left(P_{1}-P_{2}\right)\left(P_{3}-P_{4}\right)\left(P_{5}-P_{6}\right)}{\left(P_{2}-P_{3}\right)\left(P_{4}-P_{5}\right)\left(P_{6}-P_{1}\right)}=-1
$$


on the complex plane. In this section, we focus on its derivation in the context of superposition principles associated with the Kadomtsev-Petviashvili (KP) hierarchy and quasi-conformal mappings.

\subsection{The Schwarzian KP hierarchy}

The Schwarzian KP (SKP) equation

$$
\Phi_{t_{3}}=\Phi_{t_{1} t_{1} t_{1}}+\frac{3}{2} \frac{\Phi_{t_{2}}^{2}-\Phi_{t_{1} t_{1}}^{2}}{\Phi_{t_{1}}}+3 W_{t_{2}} \Phi_{t_{1}}, \quad W_{t_{1}}=\frac{\Phi_{t_{2}}}{\Phi_{t_{1}}}
$$

arises within the Painlevé analysis as the singularity manifold equation associated with the KP equation [11]. Here, $\Phi\left(t_{1}, t_{2}, t_{3}\right)$ denotes a complex-valued function and $\Phi_{t_{i}}=\partial \Phi / \partial t_{i}$. The SKP equation is readily seen to be invariant under the class of Möbius transformations

$$
\Phi \rightarrow \Phi^{\prime}=\frac{a \Phi+b}{c \Phi+d}
$$

where $a, b, c, d$ are arbitrary complex constants $(a d-b c \neq 0)$, if one formulates (2.2) in terms of the Schwarzian derivative [11]. It constitutes the first member of the infinite Möbius invariant SKP hierarchy of integrable equations with independent variables $t_{1}, t_{2}, t_{3}, t_{4}, \ldots$. The latter hierarchy admits a purely algebraic superposition formula which has been set down in [12, 13]. Thus, if

$$
\Phi=\Phi(t), \quad t=\left(t_{1}, t_{2}, t_{3}, \ldots\right)
$$

constitutes a solution of the SKP hierarchy then the six solutions

$$
\Phi_{i}=T_{i} \Phi, \quad \Phi_{i k}=T_{i} T_{k} \Phi, \quad i, k=1,2,3 ; \quad i \neq k,
$$

where

$$
T_{i} \Phi(t)=\Phi\left(t+\left[a_{i}\right]\right)=\Phi\left(t_{1}+a_{i}, t_{2}+\frac{a_{i}^{2}}{2}, t_{3}+\frac{a_{i}^{3}}{3}, \ldots\right)
$$

and $a_{i}=$ const, obey the 6-point relation (2.1) in the form

$$
\frac{\left(\Phi_{1}-\Phi_{12}\right)\left(\Phi_{2}-\Phi_{23}\right)\left(\Phi_{3}-\Phi_{13}\right)}{\left(\Phi_{12}-\Phi_{2}\right)\left(\Phi_{23}-\Phi_{3}\right)\left(\Phi_{13}-\Phi_{1}\right)}=-1 .
$$

In the present context, the relation (2.7) may be interpreted as a lattice equation if one introduces the change of variables [14]

$$
t_{k}=\frac{1}{k} \sum_{l=1}^{\infty} a_{l}^{k} n_{l}, \quad k=1,2,3, \ldots
$$

so that the operations $T_{i}, i=1,2,3$ are associated with unit increments of the variables $n_{i}$, that is, for instance,

$$
T_{1} \Phi\left(n_{1}, n_{2}, n_{3}, \ldots\right)=\Phi\left(n_{1}+1, n_{2}, n_{3}, \ldots\right) .
$$


In fact, it has been shown [12, 13] that (2.7) constitutes a discrete version of the SKP hierarchy and may therefore be termed discrete Schwarzian KP (dSKP) equation. The dSKP equation not only encodes the SKP hierarchy but also its Bäcklund transformation and various semi-discrete hierarchies 12, 13, 15. It is noted that, by construction, the dSKP equation is invariant under both the Möbius transformation (2.3) and the SKP flows.

The relation between $\Phi$ and the KP wave function $f$ and its dual $f^{*}$ is given by $12,13,15$

$$
\Delta_{i} \Phi=f^{*} f_{i}
$$

where $\Delta_{i}=T_{i}-1, f_{i}=T_{i} f$ and $f, f^{*}$ are related by

$$
\frac{f_{i}-f_{k}}{f_{i k}}=\frac{f_{k}^{*}-f_{i}^{*}}{f^{*}} .
$$

Elimination of $f$ or $f^{*}$ gives rise to the 'discrete (dual) wave function equations'

$$
\begin{array}{r}
\frac{f_{1}-f_{2}}{f_{12}}+\frac{f_{2}-f_{3}}{f_{23}}+\frac{f_{3}-f_{1}}{f_{13}}=0 \\
\frac{f_{13}^{*}-f_{12}^{*}}{f_{1}^{*}}+\frac{f_{12}^{*}-f_{23}^{*}}{f_{2}^{*}}+\frac{f_{23}^{*}-f_{13}^{*}}{f_{3}^{*}}=0 .
\end{array}
$$

In fact, it is not difficult to show that the dSKP equation guarantees that there exists a parametrization of the form $(2.10)$ and the compatibility conditions $\left[\Delta_{i}, \Delta_{k}\right] \Phi=0$ result in (2.11). In addition, the compatibility conditions associated with the relations

$$
\frac{\Delta_{i} \Phi}{\Delta_{k} \Phi}=\frac{f_{i}}{f_{k}}, \quad \frac{\Delta_{i}^{*} \Phi}{\Delta_{k}^{*} \Phi}=\frac{f_{\bar{i}}^{*}}{f_{\bar{k}}^{*}},
$$

where $f_{\bar{i}}=T_{i}^{-1} f$ and $\Delta_{i}^{*}=T_{i}^{-1}-1$, produce (2.12). Conversely, if $f$ and $f^{*}$ are solutions of (2.12) related by (2.11) then the defining relations (2.10) for $\Phi$ are compatible and the dSKP equation is satisfied. The algebraic superposition formulae (2.12) for the discrete (dual) KP wave functions have been derived in a different manner in 16 .

\subsection{A superposition principle associated with the classical Darboux transformation}

A generic feature of integrable systems is the existence of superposition principles (permutability theorems) associated with Bäcklund-Darboux-type transformations [17]. It turns out that the dSKP equation may also be derived via iterative application of the classical Darboux transformation [18] to the 'scattering problem' of the KP hierarchy

$$
\phi_{y}=\phi_{x x}+u \phi,
$$


where $x=t_{1}, y=t_{2}$. Thus, if $\phi$ and $\phi^{i}, i=1,2,3$ are solutions of the Schrödinger equation (2.14) corresponding to the potential $u$ then another three solutions $\left(\phi_{i}, u_{i}\right)$ are given by the Darboux transforms

$$
\mathbb{D}_{i}: \quad \phi_{i}=\phi_{x}-\frac{\phi_{x}^{i}}{\phi^{i}} \phi, \quad u_{i}=u+2\left(\ln \phi^{i}\right)_{x x} .
$$

The particular solutions $\left(\phi_{i}^{k}, u_{i}\right)$ defined by

$$
\phi_{i}^{k}=\phi_{x}^{k}-\frac{\phi_{x}^{i}}{\phi^{i}} \phi^{k}, \quad i \neq k
$$

may be used to obtain the 'second-generation' Darboux transforms

$$
\phi_{i k}=\phi_{i x}-\frac{\phi_{i x}^{k}}{\phi_{i}^{k}} \phi_{i}=\frac{\left|\begin{array}{ccc}
\phi^{i} & \phi_{x}^{i} & \phi_{x x}^{i} \\
\phi^{k} & \phi_{x}^{k} & \phi_{x x}^{k} \\
\phi & \phi_{x} & \phi_{x x}
\end{array}\right|}{\left|\begin{array}{ll}
\phi^{i} & \phi_{x}^{i} \\
\phi^{k} & \phi_{x}^{k}
\end{array}\right|} .
$$

The latter formulation in terms of Wronskians shows that $\phi_{i k}=\phi_{k i}$ which encapsulates the well-known permutability theorem associated with the classical Darboux transformation:

$$
\mathbb{D}_{i} \circ \mathbb{D}_{k}=\mathbb{D}_{k} \circ \mathbb{D}_{i}
$$

Due to this important commutativity property, it is possible to interpret the action of the Darboux transformations $\mathbb{D}_{i}$ as shifts on a lattice, that is, for instance,

$$
\phi=\phi\left(n_{1}, n_{2}, n_{3}\right), \quad \phi_{1}=\phi\left(n_{1}+1, n_{2}, n_{3}\right), \quad \phi_{12}=\phi\left(n_{1}+1, n_{2}+1, n_{3}\right) .
$$

It is now readily verified that the relations $(2.15)_{1}$ and 2.17 may be combined to obtain the superposition principle (or lattice equation)

$$
\frac{\phi_{13}-\phi_{12}}{\phi_{1}}+\frac{\phi_{12}-\phi_{23}}{\phi_{2}}+\frac{\phi_{23}-\phi_{13}}{\phi_{3}}=0 .
$$

The latter is precisely of the form $(2.12)_{2}$.

In order to derive the dSKP equation, it is required to take into account the action of the Darboux transformation on the adjoint Schrödinger equation

$$
-\psi_{y}=\psi_{x x}+u \psi
$$

and the associated bilinear potential $M=M(\psi, \phi)$ defined by

$$
M_{x}=\psi \phi, \quad M_{y}=\psi \phi_{x}-\psi_{x} \phi .
$$

Thus, it is readily verified that the pairs $\left(\psi_{i}, u_{i}\right)$, where

$$
\psi_{i}=-\frac{M^{i}}{\phi^{i}}, \quad M^{i}=M\left(\psi, \phi^{i}\right)
$$


constitute solutions of the adjoint Schrödinger equation (2.21). Moreover, the bilinear potentials $M_{i}=M\left(\psi_{i}, \phi_{i}\right)$ read

$$
M_{i}=M-M^{i} \frac{\phi}{\phi^{i}},
$$

where the constants of integration have been set to zero. In particular, the bilinear potentials $M_{i}^{k}=M\left(\psi_{i}, \phi_{i}^{k}\right)$ are given by

$$
M_{i}^{k}=M^{k}-M^{i} \frac{\phi^{k}}{\phi^{i}} .
$$

Accordingly, the second-generation bilinear potentials $M_{i k}=M\left(\psi_{i k}, \phi_{i k}\right)$ assume the form

$$
M_{i k}=M_{i}-M_{i}^{k} \frac{\phi_{i}}{\phi_{i}^{k}}=M+\frac{M^{i} \phi^{k} \phi_{k}-M^{k} \phi^{i} \phi_{i}}{\phi^{i} \phi_{x}^{k}-\phi^{k} \phi_{x}^{i}}
$$

so that, once again, $M_{i k}=M_{k i}$. Elimination of the quantitites $M_{i}, \phi_{i}$ and $\phi_{x}^{i}$ from (2.24) and (2.26) now produces the superposition principle

$$
\frac{\left(M_{1}-M_{12}\right)\left(M_{2}-M_{23}\right)\left(M_{3}-M_{13}\right)}{\left(M_{12}-M_{2}\right)\left(M_{23}-M_{3}\right)\left(M_{13}-M_{1}\right)}=-1
$$

which, regarded as a lattice equation, is nothing but the dSKP equation.

\subsection{A superposition principle for quasi-conformal map- pings}

In Section 6, it is shown that, in the continuum limit, the solutions of the dSKP equation define particular quasi-conformal mappings. The latter constitute mappings of the form

$$
Z: \mathbb{C} \rightarrow \mathbb{C}
$$

defined by

$$
Z_{\bar{z}}=\mu Z_{z}
$$

where $\mu$ denotes a bounded but otherwise arbitrary function. If $\mu=0$ then $Z_{\bar{z}}=0$ and hence $Z$ is analytic and defines a conformal mapping. In general, $Z$ is termed a pseudo-analytic function. Quasi-conformal mappings have various important applications in mathematics and physics [19]- 23]. In particular, they have been used in the study of plane gasdynamics by Bers 24.

The Beltrami equation (2.29) is invariant under the Darboux-type transformations

$$
\mathbb{D}_{i}: \quad Z \rightarrow Z_{i}=Z-\frac{Z^{i}}{Z_{z}^{i}} Z_{z},
$$

where $Z^{i}, i=1,2,3$ represent another three solutions of the Beltrami equation with the same potential $\mu$. It is noted that $\mu$ is preserved by $\mathbb{D}_{i}$. As in Section 2.2, the particular solutions

$$
Z_{i}^{k}=Z^{k}-\frac{Z^{i}}{Z_{z}^{i}} Z_{z}^{k}, \quad i \neq k
$$


generate the Darboux transforms

$$
Z_{i k}=Z_{i}-\frac{Z_{i}^{k}}{Z_{i z}^{k}} Z_{i z}
$$

Thus, the commutativity property $Z_{i k}=Z_{k i}$ holds and, as a consequence, the six relations (2.32) may be combined to obtain

$$
\frac{\left(Z_{1}-Z_{12}\right)\left(Z_{2}-Z_{23}\right)\left(Z_{3}-Z_{13}\right)}{\left(Z_{12}-Z_{2}\right)\left(Z_{23}-Z_{3}\right)\left(Z_{13}-Z_{1}\right)}=-1
$$

Accordingly, the dSKP equation may also be regarded as a superposition principle for quasi-conformal mappings or pseudo-analytic functions.

\subsection{Tau-function parametrizations}

It is well-known (see, e.g., [6]) that the KP hierarchy and associated quantities may be expressed in terms of tau-functions which obey the discrete Hirota equation

$$
\tau_{12} \tau_{3}=\tau_{23} \tau_{1}+\tau_{13} \tau_{2}
$$

For instance, the dual wave function $f^{*}$ is related to a tau-function $\tau$ via

$$
f_{2}^{*}=f_{1}^{*}+\frac{\tau_{12} \tau}{\tau_{1} \tau_{2}} f^{*}, \quad f_{3}^{*}=f_{1}^{*}+\frac{\tau_{13} \tau}{\tau_{1} \tau_{3}} f^{*} .
$$

This linear system is compatible modulo the discrete Hirota equation and the discrete dual wave function equation $(2.12)_{2}$ is identically satisfied.

Now, on the one hand, the quantity

$$
\tilde{\tau}=f^{*} \tau
$$

is readily shown to be another solution of the discrete Hirota equation. In fact, $\tilde{\tau}$ constitutes a discrete Darboux transform of $\tau$. Thus, the dual KP wave function admits the representation

$$
f^{*}=\frac{\tilde{\tau}}{\tau},
$$

where $\tilde{\tau}$ is a solution of the compatible system

$$
\begin{aligned}
& \tilde{\tau}_{2} \tau_{1}=\tilde{\tau}_{1} \tau_{2}+\tilde{\tau} \tau_{12} \\
& \tilde{\tau}_{3} \tau_{1}=\tilde{\tau}_{1} \tau_{3}+\tilde{\tau} \tau_{13} \\
& \tilde{\tau}_{2} \tau_{3}=\tilde{\tau}_{3} \tau_{2}+\tilde{\tau} \tau_{23} .
\end{aligned}
$$

The equations $(2.38)_{1,2}$ are obtained by inserting $f^{*}$ as given by $(2.37)$ into the linear system $(2.35)$. The remaining relation $(2.38)_{3}$ is redundant and has been added merely for reasons of symmetry.

On the other hand, the quantity

$$
\hat{\tau}=\Phi \tau
$$


also constitutes another solution of the discrete Hirota equation. This solution may be interpreted as a discrete binary Darboux transform of the seed solution $\tau$. Accordingly, any solution of the dSKP equation may be written as the ratio of two tau-functions, namely

$$
\Phi=\frac{\hat{\tau}}{\tau}
$$

In conclusion, it is noted that the integrable nature of the dSKP equation (2.7) and the discrete (dual) KP wave function equations (2.12) is inherited from the integrable (Schwarzian) KP hierarchy. In particular, the IST and $\bar{\partial}$-dressing methods and Bäcklund transformations may be employed to construct large classes of corresponding solutions [3, 田, 5, 17]. On the other hand, the fact that the dSKP equation resembles some equations of integrable discrete geometry 25] and is invariant under Möbius transformations suggests that the dSKP equation may be interpreted geometrically. Such belief has been expressed in [15] but no satisfactory geometric interpretation has been provided. Here, we address this problem and place the dSKP equation in a rather unexpected geometric setting.

\section{Menelaus' theorem, Clifford configurations and the local dSKP equation}

In the preceding, it has been shown that the 6-point relation (2.1) may be identified with the local dSKP equation (2.7). Now, the remarkable observation is that this 6-point relation encapsulates nothing but a theorem which was set down some 2000 years ago and 'is of great importance' [26] in plane geometry (see, e.g., [27, 28]). It bears the name of Menelaus and may be regarded as an axiom of affine geometry [29].

\subsection{Menelaus' theorem}

Theorem 1 (Menelaus' theorem $\left[\mathrm{I}^{\text {st }}\right.$ century AD] and its converse). Let $A, B, C$ be the vertices of a triangle and $D, E, F$ be three points on the

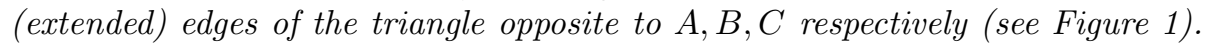
Then, the points $D, E, F$ are collinear if and only if

$$
\overline{\overline{F B}} \frac{\overline{B D}}{\overline{D C}} \frac{\overline{C E}}{\overline{E A}}=-1,
$$

where $\overline{P Q} / \overline{Q R}$ denotes the ratio of directed lengths associated with any three collinear points $P, Q, R$.

The key relation (3.1) is indeed of the form (2.1) or (2.7) but it is formulated in terms of real numbers. Its complex-valued version (see, e.g., [30]) is obtained by regarding the plane spanned by the triangle as the complex plane and labelling the points $A, \ldots, F$ by complex numbers according to (see Figure 11)

$$
A=\Phi_{1}, \quad B=\Phi_{2}, \quad C=\Phi_{3}, \quad D=\Phi_{23}, \quad E=\Phi_{13}, \quad F=\Phi_{12} .
$$



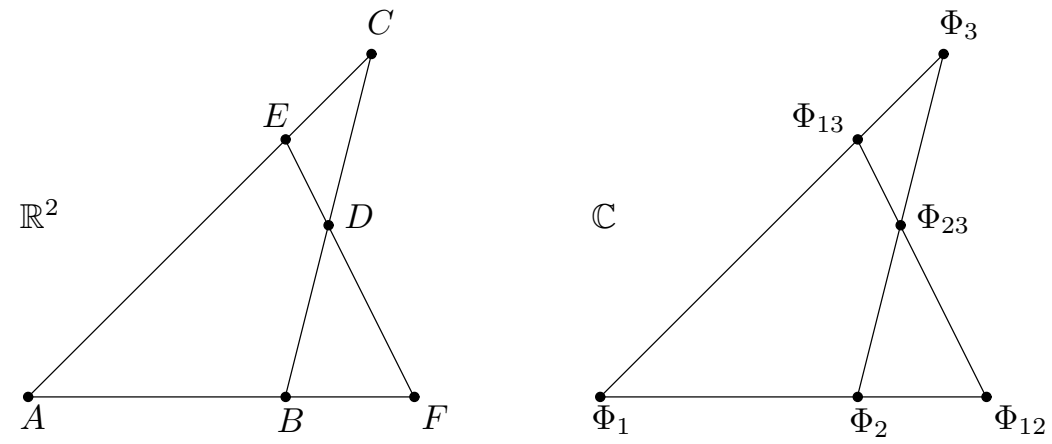

Figure 1: A Menelaus figure

Due to the collinearity of the points $(A, B, F),(B, D, C)$ and $(A, E, C)$, the ratios

$$
\alpha=\frac{\Phi_{12}-\Phi_{1}}{\Phi_{12}-\Phi_{2}}, \quad \beta=\frac{\Phi_{23}-\Phi_{2}}{\Phi_{23}-\Phi_{3}}, \quad \gamma=\frac{\Phi_{13}-\Phi_{3}}{\Phi_{13}-\Phi_{1}}
$$

are real and, indeed,

$$
\alpha=-\frac{\overline{A F}}{\overline{F B}}, \quad \beta=-\frac{\overline{B D}}{\overline{D C}}, \quad \gamma=-\frac{\overline{C E}}{\overline{E A}} .
$$

Thus, if we define the multi-ratio

$$
M\left(P_{1}, P_{2}, P_{3}, P_{4}, P_{5}, P_{6}\right)=\frac{\left(P_{1}-P_{2}\right)\left(P_{3}-P_{4}\right)\left(P_{5}-P_{6}\right)}{\left(P_{2}-P_{3}\right)\left(P_{4}-P_{5}\right)\left(P_{6}-P_{1}\right)}
$$

then the Menelaus relation (3.1) may be cast into the form

$$
M\left(\Phi_{1}, \Phi_{12}, \Phi_{2}, \Phi_{23}, \Phi_{3}, \Phi_{13}\right)=-1 .
$$

We therefore conclude that any six points associated with a 'Menelaus figure' (Figure 1) give rise to a particular solution of the local dSKP equation.

The general solution of (3.6) is obtained by exploiting its symmetry group. It is evident that, in addition to the Möbius transformation (2.3), equation (3.6) is invariant under complex conjugation $\Phi \rightarrow \bar{\Phi}$. The set of these transformations form the group of inversive transformations on the plane. It consists of Euclidean motions, scalings and inversions in circles. Analytically, the latter are represented by transformations of the form

$$
\Phi \rightarrow \Phi^{\prime}=\Phi_{*}+\frac{r^{2}}{\overline{\Phi-\bar{\Phi}_{*}}},
$$

where $r$ and $\Phi_{*}$ denote the radius and centre of a circle respectively. The inversion (3.7) converts circles passing through the point $\Phi_{*}$ into straight lines and vice versa while generic circles are mapped to circles. Inversive geometry 
is concerned with the properties of figures on the plane which are preserved by inversive transformations 28, 31]. Accordingly, the 6-point relation (3.6) and therefore the dSKP equation are objects of inversive geometry on the plane.

If we now apply a generic inversion to a Menelaus figure (Figure i1) then we obtain four circles passing through the point $\Phi_{*}$ as shown in Figure 2. It

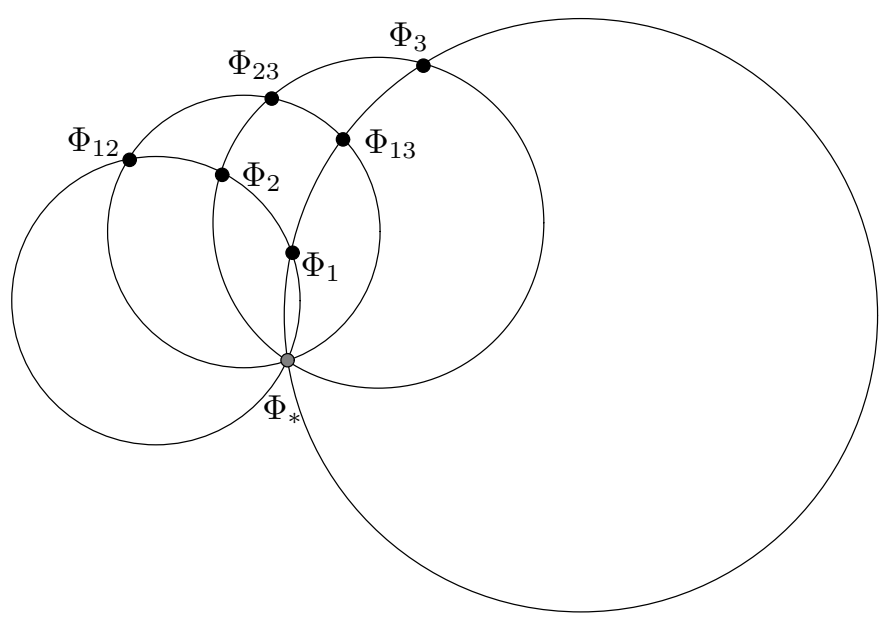

Figure 2: A Menelaus configuration

is natural to term this figure 'Menelaus configuration' since it may be mapped to a Menelaus figure by means of an inversion with respect to the point of intersection $\Phi_{*}$ without changing the multi-ratio $M\left(\Phi_{1}, \Phi_{12}, \Phi_{2}, \Phi_{23}, \Phi_{3}, \Phi_{13}\right)$. A simple counting argument indicates that the set of Menelaus configurations should encapsulate the general solution of (3.6). Indeed, the following theorem which constitutes a geometric interpretation of the 6 -point relation (3.6) holds:

Theorem 2 (Inversive geometry of the local dSKP equation). Let $\Phi_{1}, \Phi_{12}, \Phi_{2}, \Phi_{23}, \Phi_{3}, \Phi_{13}$ be six generic points on the complex plane. Then, the four circles passing through $\left(\Phi_{1}, \Phi_{12}, \Phi_{2}\right),\left(\Phi_{2}, \Phi_{23}, \Phi_{3}\right),\left(\Phi_{3}, \Phi_{13}, \Phi_{1}\right)$ and $\left(\Phi_{12}, \Phi_{23}, \Phi_{13}\right)$ meet at a point $\Phi_{*}$ if and only if

$$
M\left(\Phi_{1}, \Phi_{12}, \Phi_{2}, \Phi_{23}, \Phi_{3}, \Phi_{13}\right)=-1
$$

Proof. On the one hand, if the four circles intersect at a point $\Phi_{*}$ then one may apply an inversion with respect to that point and a Menelaus figure is obtained. According to the Menelaus theorem, the associated multi-ratio is equal to -1 and since the multi-ratio is invariant under inversive transformations (up to complex conjugation), we deduce that (3.8) holds.

On the other hand, let us assume that the 6-point relation (3.8) holds. We first draw two circles passing through the points $\left(\Phi_{1}, \Phi_{12}, \Phi_{2}\right)$ and $\left(\Phi_{3}, \Phi_{13}, \Phi_{1}\right)$. 
Since we consider the generic situation, these intersect at $\Phi_{1}$ and some point $\Phi_{*}$, say. Another two circles are now determined by the triplets $\left(\Phi_{2}, \Phi_{*}, \Phi_{3}\right)$ and $\left(\Phi_{12}, \Phi_{*}, \Phi_{13}\right)$. The latter pair of circles intersect at $\Phi_{*}$ and some point $\Phi_{\circ \circ}$. The argument employed in the first part of this proof now shows that the points $\Phi_{1}, \Phi_{12}, \Phi_{2}, \Phi_{\text {。० }}, \Phi_{3}, \Phi_{13}$ and the corresponding four circles which meet at $\Phi_{*}$ form a Menelaus configuration, whence

$$
M\left(\Phi_{1}, \Phi_{12}, \Phi_{2}, \Phi_{\circ \circ}, \Phi_{3}, \Phi_{13}\right)=-1
$$

However, since both $\Phi_{23}$ and $\Phi_{\circ \circ}$ are solutions of the same linear equation (3.8) (or (3.9) ), it is concluded that $\Phi_{\circ \circ}$ coincides with $\Phi_{23}$ and the proof is complete.

\subsection{Clifford configurations}

It is evident that if (3.8) holds then

$$
M\left(\Phi_{12}, \Phi_{2}, \Phi_{23}, \Phi_{3}, \Phi_{13}, \Phi_{1}\right)=-1 .
$$

This implies, in turn, that the four circles passing through $\left(\Phi_{13}, \Phi_{1}, \Phi_{12}\right)$, $\left(\Phi_{12}, \Phi_{2}, \Phi_{23}\right),\left(\Phi_{23}, \Phi_{3}, \Phi_{13}\right)$ and $\left(\Phi_{1}, \Phi_{2}, \Phi_{3}\right)$ likewise meet at a point $\Phi_{* *}$ as illustrated in Figure 3. Inspection of Figure 3 shows that the eight circles and

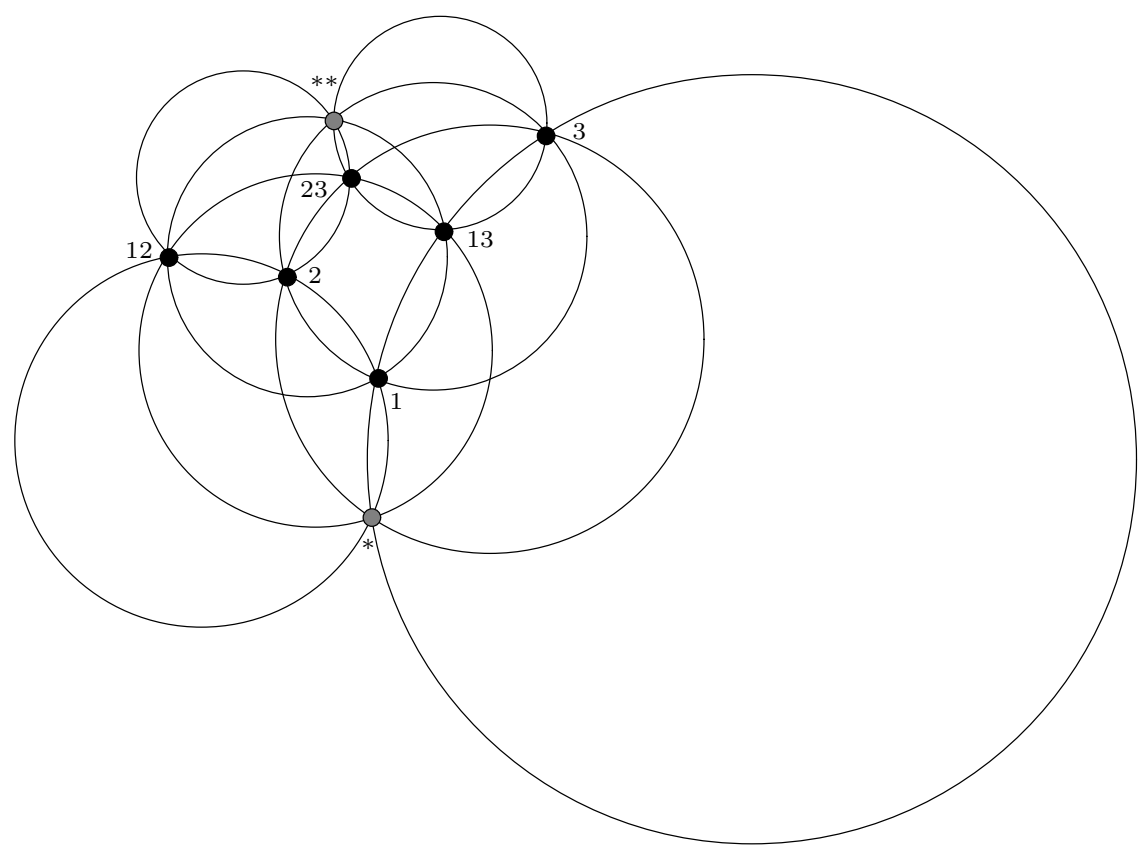

Figure 3: $\mathrm{A} \mathcal{C}_{4}$ Clifford configuration 
eight points are positioned in such a way that there exist four circles passing through each point and four points lying on each circle. Configurations of this kind are known as $\mathcal{C}_{4}$ Clifford configurations [32, 33]. These are constructed in the following way. Consider a point $P$ on the plane and four generic circles $S_{1}, S_{2}, S_{3}, S_{4}$ passing through $P$. The additional six points of intersection are labelled by $P_{12}, P_{13}, P_{14}, P_{23}, P_{24}, P_{34}$. Here, the indices on $P_{i k}$ correspond to those of the circles $S_{i}$ and $S_{k}$. By virtue of Theorem 2, the associated multi-ratio is given by

$$
M\left(P_{14}, P_{12}, P_{24}, P_{23}, P_{34}, P_{13}\right)=-1 .
$$

Any three circles $S_{i}, S_{k}, S_{l}$ intersect at three points and therefore define a circle $S_{i k l}$ passing through these points. Clifford's circle theorem 34] (and Theorem 2) then states that the four circles $S_{123}, S_{124}, S_{134}, S_{234}$ meet at a point $P_{1234}$. Thus, Figure 3 indeed displays a $\mathcal{C}_{4}$ configuration with, for instance,

$$
\begin{aligned}
& P=\Phi_{* *}, \quad P_{12}=\Phi_{12}, \quad P_{13}=\Phi_{13}, \quad P_{14}=\Phi_{1} \\
& P_{23}=\Phi_{23}, \quad P_{24}=\Phi_{2}, \quad P_{34}=\Phi_{3}, \quad P_{1234}=\Phi_{*} \text {. }
\end{aligned}
$$

Remarkably, Clifford configurations $\left(\mathcal{C}_{n}\right)$ exist for any number of initial circles $S_{1}, \ldots, S_{n}$ passing through a point $P$.

The circles and points of a $\left(\mathcal{C}_{4}\right)$ Clifford configuration are known to appear on equal footing in the sense that the angles made by the four oriented circles passing through a point are the same for all eight points [32]. Indeed, if we apply an inversion with respect to any of the eight points $P, \ldots, P_{1234}$ then four circles are mapped to four straight lines and the images of the remaining four circles meet at a point. Thus, we obtain a Menelaus figure and retrieve Wallace's theorem [35] which states that the four circumcircles of the triangles formed by four lines pass through a point. This is illustrated in Figure 4 . Due to the symmetry of the $\mathcal{C}_{4}$ configuration, corresponding lines of the eight Menelaus figures which are obtained by inversion and appropriate rotation are parallel.

It is noted in passing that there exists an elegant way of parametrizing Clifford and Menelaus configurations [31]. This may be exploited to prove in a purely algebraic manner, for instance, Steiner's theorem [36] which states that in a Wallace configuration the centres and the point of intersection of the four circumcircles lie on a circle (cf. Figure 4 ).

\section{Clifford and Menelaus lattices. Geometric in- tegrability}

In the previous section, the connection between the local dSKP equation and Menelaus configurations has been established. We now return to the interpretation of the dSKP equation

$$
M\left(\Phi_{1}, \Phi_{12}, \Phi_{2}, \Phi_{23}, \Phi_{3}, \Phi_{13}\right)=-1
$$




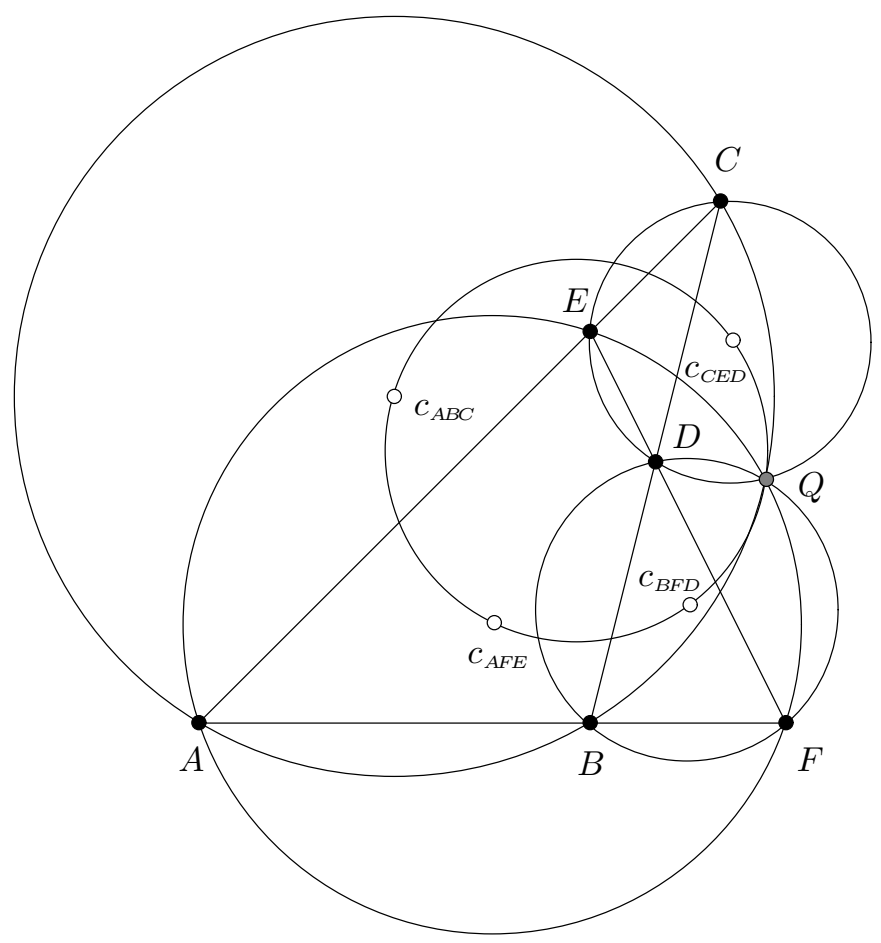

Figure 4: A Wallace configuration

as a lattice equation for maps of the form

$$
\Phi: \mathbb{Z}^{3} \rightarrow \mathbb{C} .
$$

As in Section 2, the indices on $\Phi$ are interpreted as shifts on the lattice. Accordingly, lattices of the form (4.2) subject to the dSKP equation (4.1) are integrable and consist of an infinite number of Menelaus configurations. In order to distinguish these lattices from lattices which are composed of Menelaus figures, we refer to these lattices as Clifford lattices. In this connection, it is noted that any Menelaus configuration of a Clifford lattice may be mapped to a Menelaus figure by means of an inversion. However, in general, the remaining Menelaus configurations are not converted into Menelaus figures. We here embark on a study of the 'geometric integrability' of Clifford lattices and show that Menelaus lattices indeed exist and are likewise integrable. The latter turn out to be related to a canonical reduction of the integrable discrete Darboux system [37, 38]. 


\subsection{Clifford lattices}

We begin with a lattice $\Phi$ of the form (4.2) which contains one Menelaus configuration, that is there exist six points $\Phi_{1}, \Phi_{12}, \Phi_{2}, \Phi_{23}, \Phi_{3}, \Phi_{13}$ such that (4.1) holds. It is natural to introduce complex parameters $\alpha, \beta$ and $\gamma$ according to (cf. $(3.3))$

$$
\alpha=\frac{\Phi_{12}-\Phi_{1}}{\Phi_{12}-\Phi_{2}}, \quad \beta=\frac{\Phi_{23}-\Phi_{2}}{\Phi_{23}-\Phi_{3}}, \quad \gamma=\frac{\Phi_{13}-\Phi_{3}}{\Phi_{13}-\Phi_{1}} .
$$

These encode the angles made by the edges of the triangles $\Delta\left(\Phi_{1}, \Phi_{12}, \Phi_{2}\right)$, $\Delta\left(\Phi_{2}, \Phi_{23}, \Phi_{3}\right)$ and $\Delta\left(\Phi_{3}, \Phi_{13}, \Phi_{1}\right)$ respectively and therefore the equivalence classes of triangles which are similar [30]. The definitions (4.3) may be regarded as linear equations for $\Phi_{1}, \Phi_{12}, \Phi_{2}, \Phi_{23}, \Phi_{3}, \Phi_{13}$ with the 'shape' parameters $\alpha, \beta, \gamma$ as coefficients:

$$
\begin{aligned}
& \Phi_{12}-\Phi_{1}=\alpha\left(\Phi_{12}-\Phi_{2}\right) \\
& \Phi_{23}-\Phi_{2}=\beta\left(\Phi_{23}-\Phi_{3}\right) \\
& \Phi_{13}-\Phi_{3}=\gamma\left(\Phi_{13}-\Phi_{1}\right) .
\end{aligned}
$$

The Menelaus configuration is then encapsulated in the relation

$$
\alpha \beta \gamma=1 \text {. }
$$

Shape parameters $\alpha_{3}, \beta_{1}, \gamma_{2}$ are associated with the triangles $\Delta\left(\Phi_{13}, \Phi_{123}, \Phi_{23}\right)$, $\Delta\left(\Phi_{12}, \Phi_{123}, \Phi_{13}\right)$ and $\Delta\left(\Phi_{23}, \Phi_{123}, \Phi_{12}\right)$ respectively so that

$$
\begin{aligned}
& \Phi_{123}-\Phi_{13}=\alpha_{3}\left(\Phi_{123}-\Phi_{23}\right) \\
& \Phi_{123}-\Phi_{12}=\beta_{1}\left(\Phi_{123}-\Phi_{13}\right) \\
& \Phi_{123}-\Phi_{23}=\gamma_{2}\left(\Phi_{123}-\Phi_{12}\right)
\end{aligned}
$$

which immediately implies that

$$
\alpha_{3} \beta_{1} \gamma_{2}=1 .
$$

The remaining consistency condition is obtained by eliminating $\Phi_{123}$ from any two of the relations (4.6). It turns out that, remarkably, elimination of $\Phi_{123}$ from $(4.6)_{1,2}$ leads to a constraint on the shape parameters only, namely

$$
\left(\alpha_{3} \beta_{1}-1\right)(\alpha-1)=(\alpha \beta-1)\left(\alpha_{3}-1\right) .
$$

It is emphasized that the above relation is invariant under cyclic permutation. Thus, the following theorem may be formulated:

Theorem 3 (Clifford and Menelaus lattices). A lattice $\Phi: \mathbb{Z}^{3} \rightarrow \mathbb{C}$ is composed of Menelaus configurations or figures if and only if the associated complex or real shape parameters, regarded as functions, satisfy the lattice equations

$$
\alpha \beta \gamma=1, \quad \alpha_{3} \beta_{1} \gamma_{2}=1, \quad\left(\alpha_{3} \beta_{1}-1\right)(\alpha-1)=(\alpha \beta-1)\left(\alpha_{3}-1\right) .
$$


From the point of view of integrable systems, it is natural to investigate the compatibility of the linear system (4.4) without referring to the genesis of the shape parameters. Thus, the compatibility conditions $\left(\Phi_{12}\right)_{3}=\left(\Phi_{23}\right)_{1}=\left(\Phi_{13}\right)_{2}$ lead to two equations of the form

$$
E^{1 k}\left(\Phi_{1}-\Phi_{2}\right)+E^{2 k}\left(\Phi_{2}-\Phi_{3}\right)=0, \quad k=1,2,
$$

where the coefficients $E^{i k}$ depend on the shape parameters. It turns out that these coefficients vanish if and only if the shape parameters satisfy (4.9). Hence, the 'geometric integrability' of Clifford lattices coincides with their algebraic integrability and, in fact, (4.4) is nothing but a linear triad representation of the nonlinear system (4.9) for the shape parameters. Moreover, the relations (4.9) $)_{1,2}$ imply the parametrization

$$
\alpha=\frac{\phi_{1}}{\phi_{2}}, \quad \beta=\frac{\phi_{2}}{\phi_{3}}, \quad \gamma=\frac{\phi_{3}}{\phi_{1}}
$$

in terms of some function $\phi$. The remaining relation $(4.9)_{3}$ then reads

$$
\frac{\phi_{13}-\phi_{12}}{\phi_{1}}+\frac{\phi_{12}-\phi_{23}}{\phi_{2}}+\frac{\phi_{23}-\phi_{13}}{\phi_{3}}=0
$$

which constitutes the integrable discrete dual KP wave function equation (2.12) 2 with $\phi=f^{*}$. Accordingly, the shape parameters of Clifford lattices admit the eigenfunction and tau-function representations

$$
\alpha=\frac{f_{1}^{*}}{f_{2}^{*}}=\frac{\tilde{\tau}_{1} \tau_{2}}{\tilde{\tau}_{2} \tau_{1}}, \quad \beta=\frac{f_{2}^{*}}{f_{3}^{*}}=\frac{\tilde{\tau}_{2} \tau_{3}}{\tilde{\tau}_{3} \tau_{2}}, \quad \gamma=\frac{f_{3}^{*}}{f_{1}^{*}}=\frac{\tilde{\tau}_{3} \tau_{1}}{\tilde{\tau}_{1} \tau_{3}} .
$$

It is emphasized that even though we have adopted a particular parametrization of the Menelaus figures and configurations in the construction of associated lattices, the symmetry of the Menelaus figures and configurations is still present in the sense that all lines and circles appear on equal footing. In fact, if we regard any three collinear points of a Menelaus figure as the vertices of a degenerate triangle then Menelaus lattices, that is lattices which are composed of Menelaus figures, admit the combinatorics of face-centred cubic (fcc) lattices. Indeed, if we use the reparametrization

$$
n_{4}=n_{2}+n_{3}-1, \quad n_{5}=n_{1}+n_{3}-1, \quad n_{6}=n_{1}+n_{2}-1
$$

then the lattice $\Phi$ assumes the form

$$
\begin{aligned}
\Phi: G & \rightarrow \mathbb{C} \\
G & =\left\{\left(n_{4}, n_{5}, n_{6}\right) \in \mathbb{Z}^{3}: n_{4}+n_{5}+n_{6} \text { odd }\right\} .
\end{aligned}
$$

The edge structure of $G$ induced by the edges of the 'octahedral' Menelaus figures is obtained by starting at the vertex $(1,0,0)$ and drawing diagonals across the faces of the cubic lattice $\mathbb{Z}^{3}$. Thus, $G$ is composed of octahedra and tetrahedra and constitutes an fcc lattice with $\Phi_{1}, \Phi_{12}, \Phi_{2}, \Phi_{23}, \Phi_{3}, \Phi_{13}$ or, equivalently, $\Phi_{\overline{4}}, \Phi_{6}, \Phi_{\overline{5}}, \Phi_{4}, \Phi_{\overline{6}}, \Phi_{5}$ being the vertices of the octahedra. Accordingly, the Menelaus figures (and configurations) may be regarded as the images of the octahedra under the mapping $\Phi$. 


\subsection{The Menelaus reduction of Darboux lattices}

In the preceding, it has been demonstrated that the fact that the Menelaus configuration can be extended to a Clifford lattice encodes its integrability. Here, we investigate this observation in more detail and show that the extensibility of a figure to a lattice may constitute a 'test' for integrability. Thus, for simplicity, we confine ourselves to lattices with real shape parameters. Examples of such lattices are Menelaus lattices which correspond to real solutions of the shape equations (4.9) or, equivalently, the discrete dual KP wave equation (4.12). Indeed, for any such solution, the linear system (4.4) is compatible and $M\left(\Phi_{1}, \Phi_{12}, \Phi_{2}, \Phi_{23}, \Phi_{3}, \Phi_{13}\right)=-1$.

By virtue of the Ceva theorem given below, the linear system (4.4) may likewise be associated with classical Ceva figures [27, 28] as displayed in Figure 5 .

Theorem 4 (Ceva's theorem and its converse). Let $A, B, C$ be the vertices of a triangle and $D, E, F$ be three points on the edges of the triangle opposite to $A, B, C$ respectively. Then, the lines passing through the points $(A, D),(B, E)$ and $(C, F)$ are concurrent if and only if

$$
\overline{\overline{F B}} \frac{\overline{B D}}{\overline{D C}} \frac{\overline{C E}}{\overline{E A}}=1
$$

where $\overline{P Q} / \overline{Q R}$ denotes the ratio of directed lengths associated with any three collinear points $P, Q, R$.
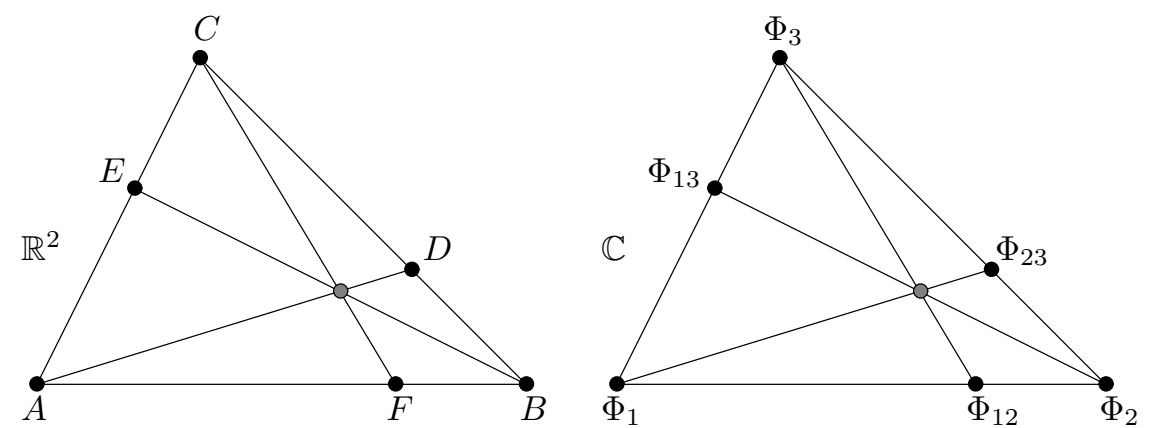

Figure 5: A Ceva figure

Indeed, if $\Phi_{1}, \Phi_{12}, \Phi_{2}, \Phi_{23}, \Phi_{3}, \Phi_{13}$ are six points defining a Ceva figure then there exist real shape parameters $\alpha, \beta, \gamma$ such that the linear system (4.4) is satisfied and

$$
M\left(\Phi_{1}, \Phi_{12}, \Phi_{2}, \Phi_{23}, \Phi_{3}, \Phi_{13}\right)=1 \quad \Leftrightarrow \quad \alpha \beta \gamma=-1 .
$$

However, if we demand that the above Ceva figure be part of a lattice with real shape parameters then the linear system (4.6) must be satisfied for some 
real shape parameters $\alpha_{3}, \beta_{1}, \gamma_{2}$. Consequently, the points $\Phi_{12}, \Phi_{23}, \Phi_{13}, \Phi_{123}$ must be collinear. This is in contradiction with the Ceva figure but consistent with the Menelaus figure. Thus, the multi-ratio $M\left(\Phi_{1}, \Phi_{12}, \Phi_{2}, \Phi_{23}, \Phi_{3}, \Phi_{13}\right)$ is required to be -1 .

It is interesting to note that the linear system (4.4), written in the form

$$
\begin{array}{ll}
\Phi_{12}=a \Phi_{1}+a^{\prime} \Phi_{2}, & a+a^{\prime}=1 \\
\Phi_{23}=b \Phi_{2}+b^{\prime} \Phi_{3}, & b+b^{\prime}=1 \\
\Phi_{13}=c \Phi_{3}+c^{\prime} \Phi_{1}, & c+c^{\prime}=1,
\end{array}
$$

has been exploited in [27] to give an algebraic proof of Menelaus' theorem, that is $a b c=-a^{\prime} b^{\prime} c^{\prime}$. Moreover, since $a, \ldots, c^{\prime}$ are real, we may think of $\Phi$ as a real two-dimensional vector-valued function satisfying (4.18) and therefore

$$
\begin{aligned}
& \Phi_{12}-\Phi=a\left(\Phi_{1}-\Phi\right)+a^{\prime}\left(\Phi_{2}-\Phi\right) \\
& \Phi_{23}-\Phi=b\left(\Phi_{2}-\Phi\right)+b^{\prime}\left(\Phi_{3}-\Phi\right) \\
& \Phi_{13}-\Phi=c\left(\Phi_{3}-\Phi\right)+c^{\prime}\left(\Phi_{1}-\Phi\right) .
\end{aligned}
$$

The latter constitutes the well-known discrete Darboux system 37, 38 on the plane if the constraints $(4.18)_{2,4,6}$ are ignored. Hence, Menelaus lattices constitute a particular reduction of Darboux lattices on the plane. In fact, if $\Phi$ is regarded as a vector in $\mathbb{R}^{n}$ then the system (4.18) is still compatible and $\Phi$ defines a conjugate lattice [25] since the (degenerate) quadrilaterals $<\Phi, \Phi_{i}, \Phi_{k}, \Phi_{i k}>, i \neq k$ are planar. Thus, the following theorem obtains:

Theorem 5 (Menelaus-Darboux lattices). The conjugate lattice $\Phi \in \mathbb{R}^{n}$ defined by the compatible linear triad

$$
\Phi_{i k}-\Phi=\frac{\phi_{k}}{\phi_{k}-\phi_{i}}\left(\Phi_{i}-\Phi\right)+\frac{\phi_{i}}{\phi_{i}-\phi_{k}}\left(\Phi_{k}-\Phi\right), \quad i \neq k
$$

where $\phi$ is a real solution of the discrete dual KP wave function equation (2.19) ${ }_{2}$, consists of (planar) Menelaus figures with vertices $\Phi_{1}, \Phi_{12}, \Phi_{2}, \Phi_{23}, \Phi_{3}, \Phi_{13}$.

\section{$5 \mathrm{KdV}$, Schramm and Boussinesq reductions}

The continuous KP equation and its hierarchy admit 1+1-dimensional reductions to the Korteweg-de Vries $(\mathrm{KdV})$ and Boussinesq (BQ) equations and their associated hierarchies. The Schwarzian analogues of these hierarchies and their integrable discretizations may be obtained from the (discrete) SKP hierarchy [39]. Here, we discuss the algebraic and geometric properties of twodimensional reductions of the dSKP equation. 


\subsection{The discrete Schwarzian KdV equation}

We first observe that the dSKP equation $M\left(\Phi_{1}, \Phi_{12}, \Phi_{2}, \Phi_{23}, \Phi_{3}, \Phi_{13}\right)=-1$ may be written as equalities of cross-ratios. For instance, we may choose the formulation

$$
Q\left(\Phi_{23}, \Phi_{1}, \Phi_{12}, \Phi_{2}\right)=Q\left(\Phi_{3}, \Phi_{13}, \Phi_{1}, \Phi_{23}\right),
$$

where the cross-ratio $Q$ of four points $P_{1}, P_{2}, P_{3}, P_{4}$ on the complex plane is defined by

$$
Q\left(P_{1}, P_{2}, P_{3}, P_{4}\right)=\frac{\left(P_{1}-P_{2}\right)\left(P_{3}-P_{4}\right)}{\left(P_{2}-P_{3}\right)\left(P_{3}-P_{4}\right)} .
$$

Thus, if we impose the constraint [39]

$$
\Phi_{23}=\Phi
$$

then the dSKP equation reduces to

$$
\Delta_{2} Q\left(\Phi, \Phi_{1}, \Phi_{12}, \Phi_{2}\right)=0
$$

whence

$$
Q\left(\Phi, \Phi_{1}, \Phi_{12}, \Phi_{2}\right)=\nu\left(n_{1}\right),
$$

where $\nu$ is an arbitrary function of $n_{1}$. If, in addition, we demand that the function $\nu$ be preserved by the group of inversive transformations then $\nu$ must be real. This corresponds to a particular case of the discrete Schwarzian KdV (dSKdV) equation 40] and implies that the points $\Phi, \Phi_{1}, \Phi_{12}, \Phi_{2}$ lie on a circle. The dSKdV equation is therefore located in the class of dSKP reductions of the type

$$
Q\left(\Phi_{23}, \Phi_{1}, \Phi_{12}, \Phi_{2}\right)=Q\left(\Phi_{3}, \Phi_{13}, \Phi_{1}, \Phi_{23}\right) \in \mathbb{R} .
$$

The latter are associated with degenerate Menelaus configurations with two touching circles as displayed in Figure 6. These two circles become parallel
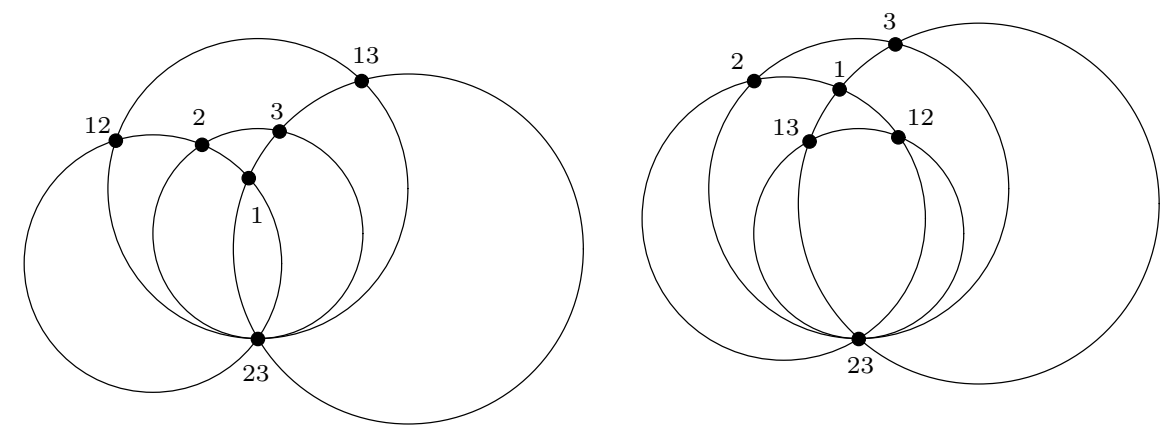

Figure 6: Degenerate Menelaus configurations

straight lines if an inversion with respect to $\Phi_{23}$ is applied. The resulting configurations as depicted in Figure 7 may be regarded as degenerate Menelaus 

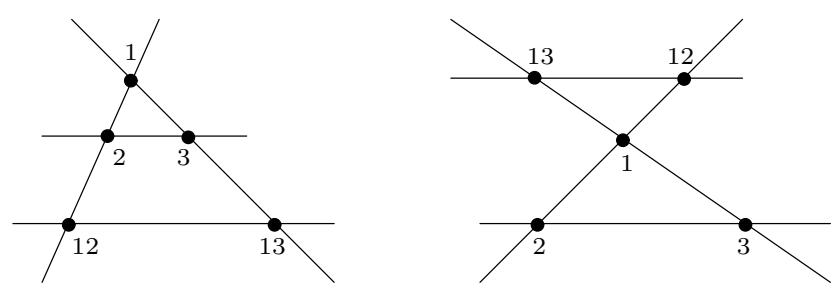

Figure 7: Degenerate Menelaus figures

figures. It is also noted that since $\nu$ is independent of $n_{2}$, the relation (5.5) defines particular generalized discrete conformal mappings as introduced in 441. The case $\mu=-1$ corresponds to the standard definition of discrete conformal mappings [41]. Accordingly, discrete conformal mappings give rise to special degenerate Clifford lattices and their constituent Menelaus and $\mathcal{C}_{4}$ Clifford configurations.

\subsection{P lattices and Schramm's circle patterns}

Further degeneration of the Menelaus configuration leads to two pairs of touching circles. Indeed, if two points with complementary indices coincide, that is, for instance,

$$
\Phi_{3}=\Phi_{12},
$$

then a Menelaus configuration of the type displayed in Figure 8 is obtained. In
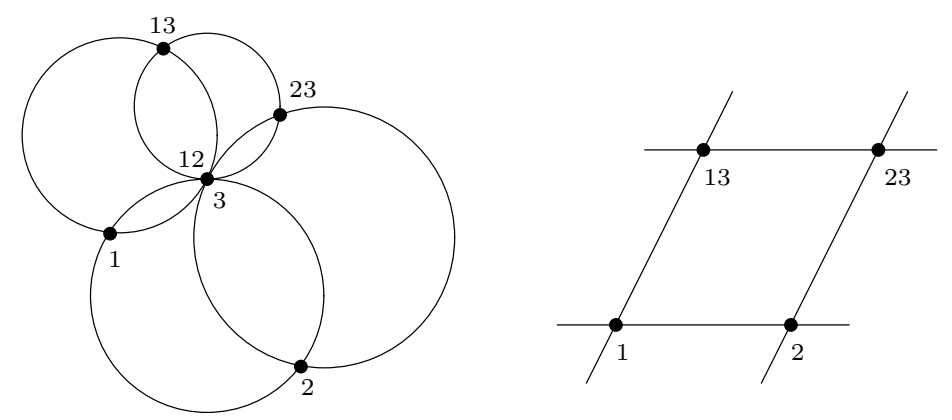

Figure 8: A 5-point Menelaus configuration and its image under inversion

fact, five points $\Phi_{1}, \Phi_{12}, \Phi_{2}, \Phi_{23}, \Phi_{13}$ on the complex plane obey the multi-ratio condition

$$
M\left(\Phi_{1}, \Phi_{12}, \Phi_{2}, \Phi_{23}, \Phi_{12}, \Phi_{13}\right)=-1
$$

if and only if the pairs of 'opposite' circles $S\left(\Phi_{1}, \Phi_{12}, \Phi_{2}\right), S\left(\Phi_{23}, \Phi_{12}, \Phi_{13}\right)$ and $S\left(\Phi_{12}, \Phi_{2}, \Phi_{23}\right), S\left(\Phi_{12}, \Phi_{13}, \Phi_{1}\right)$ are tangent. In this case, inversion with respect to $\Phi_{12}$ leads to two pairs of parallel straight lines (see Figure 8). Thus, if we 
impose the condition (5.7) on a Clifford lattice then the dSKP equation reduces to (5.8) or, equivalently,

$$
\frac{\left(\Phi_{\overline{2}}-\Phi\right)\left(\Phi_{\overline{1}}-\Phi_{2}\right)\left(\Phi-\Phi_{1}\right)}{\left(\Phi-\Phi_{\overline{1}}\right)\left(\Phi_{2}-\Phi\right)\left(\Phi_{1}-\Phi_{\overline{2}}\right)}=-1
$$

and the geometry of the corresponding two-dimensional lattice is characterized by the property that any vertex $\Phi$ and its neighbours $\Phi_{1}, \Phi_{2}, \Phi_{\overline{1}}, \Phi_{\overline{2}}$ are positioned in such a way that opposite circles through these points are tangent. Since inversion with respect to any given vertex $\Phi$ produces a parallelogram, lattices of this kind are termed $P$ lattices 42]. It is known that the assumption that the elementary quadrilaterals are inscribed in circles which intersect orthogonally is admissible and preserves integrability [42]. Moreover, if the elementary quadrilaterals are embedded then Schramm's important circle patterns are retrieved [43]. These may be used to approximate analytic functions (see 44).

\subsection{The discrete Schwarzian Boussinesq equation}

The reduction 39]

$$
\Phi_{123}=\Phi
$$

leads to the two-dimensional lattice equation $M\left(\Phi_{1}, \Phi_{12}, \Phi_{2}, \Phi_{\overline{1}}, \Phi_{\overline{1} \overline{2}}, \Phi_{\overline{2}}\right)=-1$, that is

$$
\frac{\left(\Phi_{1}-\Phi_{12}\right)\left(\Phi_{2}-\Phi_{\overline{1}}\right)\left(\Phi_{\overline{1} \overline{2}}-\Phi_{\overline{2}}\right)}{\left(\Phi_{12}-\Phi_{2}\right)\left(\Phi_{\overline{1}}-\Phi_{\overline{1} \overline{2}}\right)\left(\Phi_{\overline{2}}-\Phi_{1}\right)}=-1
$$

which is known as the discrete Schwarzian Boussinesq (dSBQ) equation [45]. dSBQ lattices are therefore defined by the property that any sextuplet $\left(\Phi_{1}, \Phi_{12}, \Phi_{2}, \Phi_{\overline{1}}, \Phi_{\overline{1}}, \Phi_{\overline{2}}\right)$ represents a Menelaus configuration. Any six points of this kind constitute the vertices of a hexagon if we extend the square lattice to a triangular lattice by introducing one family of diagonals as indicated in Figure 9. Since triangular lattices may be covered by three overlapping hexagonal sublattices, dSBQ lattices may also be interpreted as triangular lattices which are such that the three honeycomb lattices are composed of Menelaus configurations. These triangular lattices and their concomitant hexagonal lattices have recently been introduced and discussed in detail in 446 in the context of hexagonal circle patterns. The Menelaus connection presented here therefore provides a geometric interpretation of these lattices and, by construction, their integrability is inherited from Clifford lattices. A geometric description of the multi-ratio condition (5.11) in the case of six concyclic points has been given earlier in 47.

In conclusion, it is noted that there exist other canonical integrable reductions of the dSKP equation such as periodic fixed point reductions and symmetry reductions associated with the group of inversive transformations. For instance, we may assume that a Clifford lattice is invariant under inversion in a circle which is equivalent to considering Clifford lattices on a circle. Thus, if we focus on the unit circle centred at the origin then the parametrization

$$
\Phi=e^{2 \mathrm{i} \omega}
$$




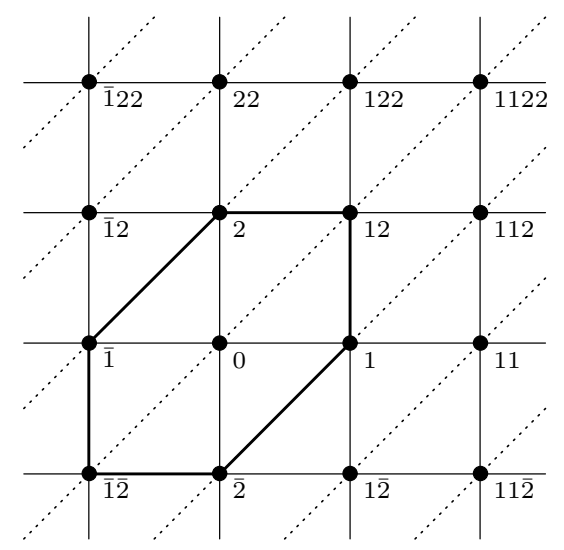

Figure 9: The dSBQ grid

reduces the dSKP equation to

$$
\frac{\sin \left(\omega_{1}-\omega_{12}\right) \sin \left(\omega_{2}-\omega_{23}\right) \sin \left(\omega_{3}-\omega_{13}\right)}{\sin \left(\omega_{12}-\omega_{2}\right) \sin \left(\omega_{23}-\omega_{3}\right) \sin \left(\omega_{13}-\omega_{1}\right)}=-1 .
$$

The corresponding 'circular' lattices may be mapped to the complex plane by means of a Combescure-type transformation which, in fact, exists for any Clifford lattice. Indeed, it is readily verified that if $\Phi$ constitutes a solution of the dSKP equation with corresponding shape parameters $\alpha, \beta, \gamma$ then the linear system

$$
\begin{aligned}
& \rho_{2}-\rho=\alpha\left(\rho_{1}-\rho\right) \\
& \rho_{3}-\rho=\beta\left(\rho_{2}-\rho\right) \\
& \rho_{1}-\rho=\gamma\left(\rho_{3}-\rho\right)
\end{aligned}
$$

is compatible. The compatibility of this linear system which may be regarded as adjoint to (4.4) guarantees that there exists a bilinear potential $\Phi^{\prime}$ defined by

$$
\Phi_{i}^{\prime}-\Phi^{\prime}=\rho\left(\Phi_{i}-\Phi\right), \quad i=1,2,3 .
$$

It is readily verified that $\Phi^{\prime}$ constitutes another solution of the dSKP equation.

\section{The natural continuum limit. (Quasi-)confor- mal mappings}

The standard continuum limit of the dSKP equation represented by $\Phi_{i}(t)=$ $\Phi\left(t+\left[a_{i}\right]\right), a_{i} \rightarrow 0$ gives rise to the SKP hierarchy [12, 13, 15]. However, from a geometric point of view, it is natural to consider the limit in which the polygons $\Phi\left(n_{i}=\right.$ const, $n_{k}=$ const $)$ become coordinate lines on the complex plane. Thus, 
we regard the differences $\Delta_{i} \Phi=\Phi_{i}-\Phi$ between two neighbouring points $\Phi_{i}$ and $\Phi$ as approximations of derivatives, that is

$$
\Phi_{i}=\Phi+\epsilon \Phi_{x_{i}}+O\left(\epsilon^{2}\right), \quad i=1,2,3,
$$

where $\epsilon$ is a lattice parameter and $\Phi_{x_{i}}=\partial \Phi / \partial x_{i}$. In the limit $\epsilon \rightarrow 0$ and $\left(x_{1}, x_{2}, x_{3}\right)=(x, y, t)$, the dSKP then reduces to

$$
\left(\ln \frac{\Phi_{x}}{\Phi_{y}}\right)_{t}+\left(\ln \frac{\Phi_{y}}{\Phi_{t}}\right)_{x}+\left(\ln \frac{\Phi_{t}}{\Phi_{x}}\right)_{y}=0
$$

while the linear system (4.4) reads

$$
\Phi_{y}=\alpha \Phi_{x}, \quad \Phi_{t}=\beta \Phi_{y}, \quad \Phi_{x}=\gamma \Phi_{t} .
$$

In addition to the group of inversive transformations, equation (6.2) is invariant under $\Phi \rightarrow F(\Phi)$, where $F$ is an arbitrary differentiable function. The solutions of $(\sqrt[6.2]{ })$ constitute mappings $\Phi: \mathbb{R}^{3} \rightarrow \mathbb{C}$ to which we shall refer as SKP mappings.

As in the discrete case, the linear system (6.3) implies that the 'continuous' shape factors $\alpha, \beta, \gamma$ are constrained by $\alpha \beta \gamma=1$. Accordingly, if we set $\delta=1 / \gamma$ then SKP mappings are obtained by integrating the linear pair

$$
\Phi_{y}=\alpha \Phi_{x}, \quad \Phi_{t}=\delta \Phi_{x},
$$

where $\alpha, \delta$ are solutions of the coupled nonlinear system

$$
\alpha_{t}+\alpha \delta_{x}=\delta_{y}+\delta \alpha_{x}, \quad(1-\delta) \alpha_{t}=(1-\alpha) \delta_{y} .
$$

The latter comprises (6.2) written in terms of the shape factors and the compatibility condition for (6.4). An alternative form of (6.5) is given by the single equation

$$
\left(e^{\varphi_{y}}-e^{\varphi_{t}}\right) \varphi_{y t}+\left(1+e^{\varphi_{t}}\right) \varphi_{x y}-\left(1+e^{\varphi_{y}}\right) \varphi_{x t}=0
$$

with $\alpha=1+e^{-\varphi_{y}}, \delta=1+e^{-\varphi_{t}}$. In terms of the variables

$$
\mu=\frac{\mathrm{i}-\alpha}{\mathrm{i}+\alpha}, \quad z=x+\mathrm{i} y,
$$

the linear system (6.4) reads

$$
\Phi_{\bar{z}}=\mu \Phi_{z}, \quad \Phi_{t}=\delta\left(\Phi_{z}+\Phi_{\bar{z}}\right)
$$

so that it becomes transparent that SKP mappings are descriptive of particular evolutions of quasi-conformal mappings if the variable $t$ is interpreted as time. Here, we assume that the coefficient $\mu$ in the Beltrami equation (6.8) 1 is bounded. In other words, any SKP mapping consists of a one-parameter family $\Phi(t)$ of quasi-conformal mappings. 


\subsection{SKdV mappings}

The continuum limit of the constraint (5.3) corresponding to the dSKdV equation is readily seen to be

$$
\Phi_{y}+\Phi_{t}=0 .
$$

Accordingly, $\beta=-1$ and $\alpha=\alpha(x)$. The associated SKP mappings are then defined by the Beltrami equation

$$
\Phi_{\bar{z}}=\mu(z+\bar{z}) \Phi_{z},
$$

where $\mu$ is an arbitrary function of its argument. Thus, the SKdV mappings $\Phi(t=$ const $): \mathbb{C} \rightarrow \mathbb{C}$ constitute quasi-conformal mappings with complex dilations of the form $\mu=\mu(z+\bar{z})$. If the shape factor $\alpha$ is purely imaginary then we may assume without loss of generality that $\alpha= \pm \mathrm{i}$. This corresponds to $\mu=0$ or $\mu \rightarrow \infty$ and therefore $\Phi_{\bar{z}}=0$ or $\Phi_{z}=0$. In this way, analytic or anti-analytic functions are obtained. This is in agreement with the fact that the discrete SKdV equation (5.5) with $\nu=-1$ reduces to $\Phi_{x}^{2} / \Phi_{y}^{2}=-1$ in the continuum limit $\epsilon \rightarrow 0$ and therefore defines (anti-)analytic functions 41 .

\subsection{SBQ mappings}

The continuum limit of the constraint (5.10) corresponding to the dSBQ equation is given by

$$
\Phi_{x}+\Phi_{y}+\Phi_{t}=0 .
$$

This implies that $1+\alpha+\alpha \beta=0$ and hence the associated SKP mappings are obtained via

$$
\Phi_{\bar{z}}=\mu \Phi_{z}, \quad \mu_{\bar{z}}=\frac{1-2 \mathrm{i} \mu}{2 \mathrm{i}+\mu} \mu_{z} .
$$

Thus, the SBQ mappings $\Phi(t=$ const $): \mathbb{C} \rightarrow \mathbb{C}$ constitute quasi-conformal mappings with complex dilations $\mu$ given implicitly by

$$
\mu=G((2 \mathrm{i}+\mu) z+(1-2 \mathrm{i} \mu) \bar{z}),
$$

where $G$ is an arbitrary differentiable function.

To summarize, it has been shown that the scalar equation (6.2) and its canonical reductions define classes of 'integrable' quasi-conformal mappings. The solutions of the dSKP equation and the dSKdV and dSBQ reductions (5.5), (5.11) may therefore be regarded as their integrable discretizations.

Acknowledgement. One of the authors (B.G.K.) is grateful to the School of Mathematics, UNSW for the kind hospitality.

\section{References}

[1] B.B. Kadomtsev and V.I. Petviashvili, On the stability of solitary waves in weakly dispersive media, Dokl. Akad. Nauk SSSR 192, 753-756 (1970). 
[2] V.E. Zakharov and A.B. Shabat, A scheme for integrating the nonlinear equations of mathematical physics by the method of the inverse scattering problem I., Func. Anal. Appl. 8, 226-235 (1974).

[3] V.S. Dryuma, Analytic solutions of the two-dimensional Korteweg-de Vries equation, Soviet Phys. JETP Lett. 19, 387-388 (1974).

[4] V.E. Zakharov, S.V. Manakov, S.P. Novikov and L.P. Pitaevskii, The Theory of Solitons: The Inverse Problem Method, Nauka, Moscow (1980); Plenum Press (1984).

[5] M.J. Ablowitz and P. Clarkson, Solitons, Nonlinear Evolution Equations and Inverse Scattering, Cambridge University Press (1991).

[6] M. Jimbo and T. Miwa, Solitons and infinite dimensional Lie algebras, Publ. RIMS, Kyoto Univ. 19, 943 (1983).

[7] R. Dijkgraaf, Intersection theory, integrable hierarchies and topological field theory, in New Symmetry Principles in Quantum Field Theory, NATO ASI, Cargese (1991); Plenum Press, London (1991).

[8] A. Morozov, Integrability and matrix models, Phys. Usp. 37, 1-55 (1994).

[9] P.Di Francesco, P. Ginsparg and J. Zinn-Justin, 2D gravity and random matrices, Phys. Rep. 254, 1-133 (1995).

[10] T. Shiota, Characterization of Jacobian varieties in terms of soliton equations, Inven. Math. 83, 333-382 (1986).

[11] J. Weiss, The Painlevé property for partial differential equations. II. Bäcklund transformations, Lax pairs and Schwarzian derivative, J. Math. Phys. 24, 1405-1413 (1983).

[12] L.V. Bogdanov and B.G. Konopelchenko, Analytic-bilinear approach to integrable hierarchies. I. Generalized KP hierarchy, J. Math. Phys. 39, 4683-4700 (1998).

[13] L.V. Bogdanov and B.G. Konopelchenko, Analytic-bilinear approach to integrable hierarchies. II. Multicomponent KP and 2D Toda lattice hierarchies, J. Math. Phys. 39, 4701-4728 (1998).

[14] T. Miwa, On Hirota difference equations, Proc. Japan. Acad. A 58, 9-12 (1982).

[15] L.V. Bogdanov and B.G. Konopelchenko, Möbius invariant integrable lattice equations associated with KP and 2DTL hierarchies, Phys. Lett. A 256, 39-46 (1999).

[16] F. Nijhoff and H.W. Capel, The direct linearization approach to hierarchies of integrable PDEs in $2+1$ dimensions. I. Lattice equations and the differential-difference hierarchies, Inverse Problems 6, 567-590 (1990). 
[17] V.B. Matveev and M.A. Salle, Darboux Transformations and Solitons, Springer Verlag, Berlin (1991).

[18] G. Darboux, Sur une proposition relative aux equations lineaire, Compt. Rend. Acad. Sci. Paris 94, 1456-1459 (1882).

[19] L.V. Ahlfors, Lectures on Quasiconformal Mappings, D. Van Nostrand Company, Princeton (1966).

[20] O. Lehto and K.I. Virtanen, Quasiconformal Mappings in the Plane, Springer Verlag, New York - Heidelberg (1973).

[21] P. Caraman, n-Dimensional Quasiconformal (QCF) Mappings, Abacus Press, New Jersey (1974).

[22] Yu.G. Reshetnyak, Space mappings with bounded distorsion, Trans. Math. Monog. 73, AMS, Providence, Rhode Island (1989).

[23] M. Vuorinen, ed., Quasiconformal space mappings, Lecture Notes in Math. 1508, Springer Verlag, Berlin (1992).

[24] L. Bers, Mathematical Aspects of Subsonic and Transonic Gas Dynamics, Wiley, New York (1958).

[25] A.I. Bobenko and R. Seiler, eds., Discrete Integrable Geometry and Physics, Clarendon Press, Oxford (1999).

[26] E.H. Askwith, A Course of Pure Geometry, Cambridge University Press (1917).

[27] D. Pedoe, A Course of Geometry, Cambridge University Press (1970).

[28] D.A. Brannan, M.F. Esplen and J.J. Gray, Geometry, Cambridge University Press (1999).

[29] H. Guggenheimer, The Theorem of Menelaus in axiomatic geometry, Geometriae Dedicata 3, 257-261 (1974).

[30] J.A. Lester, Triangles II: Complex triangle coordinates, Aequationes Math. 52, 215-245 (1996).

[31] F. Morley and F.V. Morley, Inversive Geometry, Chelsea Publ. Comp., New York (1954).

[32] P. Ziegenbein, Konfigurationen in der Kreisgeometrie, J. reine angew. Math. 183, 9-24 (1941).

[33] M.S. Longuet-Higgins, Inversive properties of the plane $n$-line, and a symmetric figure of $2 \times 5$ points on a quadric, J. London Math. Soc. 12, 206-212 (1976). 
[34] W.K. Clifford, A synthetic proof of Miquel's theorem, Oxford, Cambridge and Dublin Messenger of Math. 5, 124-141 (1871).

[35] W. Wallace ("Scoticus"), Art. XXVI, in The Mathematical Repository (new series) 1, ed. T. Leybourne (1806).

[36] J. Steiner, Théorème sur le quadrilatère complet, Ann. de Math. 18, 302303 (1827).

[37] L.V. Bogdanov and B.G. Konopelchenko, Lattice and $q$-difference DarbouxZakharov-Manakov systems via $\bar{\partial}$-dressing method, J. Phys. A: Math. Gen. 28, L173-L178.

[38] A. Doliwa, Geometric discretisation of the Toda system, Phys. Lett. A 234, 187-192 (1997).

[39] L.V. Bogdanov and B.G. Konopelchenko, in preparation.

[40] F.W. Nijhoff and H.W. Capel, The discrete Korteweg-de Vries equation, Acta Appl. Math. 39, 133-158 (1995).

[41] A.I. Bobenko and U. Pinkall, Discrete isothermic surfaces, J. reine angew. Math. 475, 187-208 (1996).

[42] A.I. Bobenko and U. Pinkall, Discretization of surfaces and integrable systems, in A.I. Bobenko and R. Seiler, eds., Discrete Integrable Geometry and Physics, Clarendon Press, Oxford (1999) pp. 3-58.

[43] O. Schramm, Circle patterns with the combinatorics of the square grid, Duke Math. J. 86, 347-389 (1997).

[44] S.I. Agafonov and A.I. Bobenko, Discrete $Z^{\gamma}$ and Painlevé equations, International Math. Research Notices 4, 165-193 (2000).

[45] F.W. Nijhoff, Discrete Painlevé equations and symmetry reduction on the lattice, in A.I. Bobenko and R. Seiler, eds., Discrete Integrable Geometry and Physics, Clarendon Press, Oxford (1999) pp. 209-234.

[46] A.I. Bobenko, T. Hoffmann and Yu.B. Suris, Hexagonal circle patterns and integrable systems: Patterns with the multi-ratio property and Lax equations on the regular triangular lattice, arXiv:math.CV/0104244(2001).

[47] A.I. Bobenko and T. Hoffmann, Conformally symmetric circle packings. A generalization of Doyle spirals, Sfb 288 Preprint 468, Technische Universität Berlin (2001). 